\title{
On a Continuum Limit for Loop Quantum Cosmology
}

\author{
Alejandro Corichi, ${ }^{1,2, *}$ Tatjana Vukašinac, ${ }^{3, \oplus}$ and José Antonio Zapata, ${ }^{1,}$ \\ ${ }^{1}$ Instituto de Matemáticas, Unidad Morelia, \\ Universidad Nacional Autónoma de México, UNAM-Campus Morelia, \\ A. Postal 61-3, Morelia, Michoacán 58090, Mexico \\ ${ }^{2}$ Center for Fundamental Theory, Institute for Gravitation and the Cosmos, \\ The Pennsylvania State University, University Park PA 16802, USA \\ ${ }^{3}$ Facultad de Ingeniería Civil, Universidad Michoacana de San Nicolas de Hidalgo \\ Morelia, Michoacán 58000, Mexico
}

\begin{abstract}
The use of non-regular representations of the Heisenberg-Weyl commutation relations has proved to be useful for studying conceptual and technical issues in quantum gravity. Of particular relevance is the study of Loop Quantum Cosmology (LQC), a symmetry reduced theory that is related to Loop Quantum Gravity, and that is based on a non-regular, polymeric representation. Recently, a soluble model was used by Ashtekar, Corichi and Singh to study the relation between Loop Quantum Cosmology and the standard Wheeler-DeWitt theory and in particular the passage to the limit in which the auxiliary parameter (interpreted as "quantum geometry discreetness") is sent to zero in hope to get rid of this 'regulator ambiguity' in the LQC dynamics. In this note we outline the first steps toward reformulating this question within the program developed by the authors for studying the continuum limit of polymeric theories, which was successfully applied to simple systems such as a Simple Harmonic Oscillator and the Free Particle.
\end{abstract}

PACS numbers: 04.60.Pp, 04.60.Ds, 04.60.Nc 11.10.Gh.

*Electronic address: corichi@matmor.unam.mx

${ }^{\dagger}$ Electronic address: tatjana@shi.matmor.unam.mx

${ }^{\ddagger}$ Electronic address: zapata@matmor.unam.mx 


\section{INTRODUCTION}

Loop quantum gravity has become one of the most popular, non-stringy, approaches to quantum gravity [1]. With the aim of understanding it better a simpler, mechanical, model known as polymer quantum mechanics was developed, where a system with a finite number of degrees of freedom replaces a field theory.

The kinematical basis of polymer quantum mechanics is based on a non-regular representation of the Heisenberg-Weyl algebra (which turns out to be inequivalent to the Schrödinger's representation). Of course, von Neumann's theorem tells us that such representation must suffer from some kind of pathology. The pathology in this case is the inability of polymer quantum mechanics to capture the usual topology of the real line corresponding to the position observables [2]. This pathology is responsible for the non existence of the limits suggested by a direct quantization of the Hamiltonian (or any other observable which involves a polynomial of the momentum). In the same fashion, within full loop quantum gravity, the unique diffeo invariant quantization [3] is such that the holonomy observables which lie at its core do not enjoy of the continuity properties suggested by the topology of the space manifold, and the limits suggested by the regularization of the curvature do not exist.

A more specific motivation to better understand polymer quantum mechanics is that it is the mathematical basis of loop quantum cosmology (LQC) [4]. In complete analogy with the full theory, the polymeric representation used in LQC is such that the holonomies are well defined but the connection and curvature are not and, furthermore, the intuitive limits that one would use to define them though holonomies do not exist. The curvature is therefore defined by fixing a minimal area $\lambda^{2}$ when computing the holonomies, that is motivated by the discreetness of the quantum geometry in LQG [1].

There is a general proposal to define effective theories, coarse graining and continuum limits in a Wilsonian manner within the framework of loop quantization [5]. In this context the renormalization process modifies the continuum limit relevant for dynamics in a way that may enhance its continuity aspects. Recently the authors applied this proposal to a model of the simple harmonic oscillator in polymer quantum mechanics [6]. The resulting dynamics in the continuum limit was shown to be equivalent to that of the Schrödinger representation of the simple harmonic oscillator (compare to the original treatment of the model [2]). The continuum limit in this context is different from the kinematical continuum limit because the renormalization prescription which runs the renormalization process demands that the Hamiltonian be physically meaningful. This contribution targets specifically loop quantum cosmology, so we will direct our efforts in that direction. In particular, a natural question is whether such results can be generalized to LQC, in which case one would be interested in studying the resulting continuum limit and its relation to the Wheeler DeWitt theory.

In this contribution we will set the stage to analyze a simplified model of loop quantum cosmology introduced by Ashtekar, Corichi and Singh in [7] known as 'sLQC', from the perspective of the Wilsonian renormalization introduced in [6] to polymer quantum mechanics. In the next section we will recall the basic formalism of [6]. Later we review the solvable model of loop quantum cosmology of [7] and give a summary of what is known about the renormalization of this model. We will close our contribution with a list of open issues regarding the application of our formalism to loop quantum cosmology, we will give some remarks and some partial conclusions. 


\section{EFFECTIVE THEORIES, COARSE GRAINING AND CONTINUUM LIMIT}

In this section we define the concept of effective theories and their continuum limit. The first ingredient is the definition of a scale to which an effective theory is associated. For a detailed treatment of the subject presented in this section see [6].

The role of scales will be played by regular decompositions of $\mathbb{R}$ as a disjoint union of closed-open intervals of length $a_{n}=\frac{a_{0}}{2^{n}}$.

Definition 1 (Scale) In our context, a scale $C_{n}$ is a decomposition of the real line of the form $\mathbb{R}=\cup_{\alpha_{i} \in C_{n}} \alpha_{i}$, where $\alpha_{i}=\left[L\left(\alpha_{i}\right), R\left(\alpha_{i}\right)\right)$, and the vertex set is $\left\{L\left(\alpha_{i+1}\right)=R\left(\alpha_{i}\right)=\right.$ $\left.i a_{n}\right\}_{\alpha_{i} \in C_{n}}$.

To every scale $C_{n}$ we associate a space of states at scale $C_{n}, C y l(n)$, with a basis $\left\{e_{\alpha}\right\}_{\alpha \in C_{n}}$ labeled by the cells of $C_{n}$. The inner product is given by $\left(e_{\alpha_{i}}, e_{\alpha_{j}}\right)_{C_{n}}=\delta_{i j}$. The completion of $\operatorname{Cyl}(n)$ is the Hilbert space at the scale $C_{n}, \mathcal{H}_{n}$. This inner product is inherited from the corresponding polymer Hilbert space, see [6].

We will also work with the dual space $\mathcal{H}_{n}^{\star}$. Since the space of states at any given scale is a Hilbert space, its dual is isomorphic to it, but for us the dual space will be of special interest. Notice that its elements have a natural $\sim_{n}$-preserving action on $C y l(\mathbb{R})_{x}$ (the vector space of cylindric functions on the real line). This action is particularly simple to see for the elements of the dual basis $\left\{\omega_{\alpha}\right\}_{\alpha \in C_{n}}$; for them we have $\omega_{\alpha}\left(\delta_{x_{0}}\right)=\chi_{\alpha}\left(x_{0}\right)$ where $\delta_{x_{0}}(x)=0$ if $x \neq x_{0}$ and $\delta_{x_{0}}(x)=1$ if $x=x_{0}$, and $\chi_{\alpha}$ is the characteristic function of the set $\alpha \subset \mathbb{R}$. Thus, we write $\mathcal{H}_{n}^{\star} \subset C y l(n)^{\star}$ where by $C y l(n)^{\star}$ we mean the $\sim_{n}$-preserving subspace of $C y l(\mathbb{R})_{x}^{\star}$.

In order to define the continuum limit of the effective theories we have to relate different scales by mapping between the corresponding Hilbert spaces and its duals.

Definition 2 (Coarse graining) Given two scales we write $C_{m} \leq C_{n}$ and say that $C_{m}$ is a coarse graining of $C_{n}$ (or $C_{n}$ is a refinement of $C_{m}$ ) if any interval $\alpha_{i} \in C_{m}$ is a finite union of intervals of $C_{n}$.

Our coarse graining maps work by decimation. If we have two scales related by refinement $C_{m} \leq C_{n}$ our decimation map will be defined to be the injective isometry $d: \mathcal{H}_{m} \rightarrow \mathcal{H}_{n}$ characterized by $d\left(e_{\alpha_{i}}\right)=e_{\beta_{j}} \Longleftrightarrow L\left(\alpha_{i}\right)=L\left(\beta_{j}\right)$. It is important to notice that if $C_{m} \leq C_{n} \leq C_{o}$ the corresponding d-triangle diagram commutes. ${ }^{1}$

On the other hand, $d^{\star}: \mathcal{H}_{n}^{\star} \rightarrow \mathcal{H}_{m}^{\star}$ sends part of the elements of the dual basis to zero while keeping the information of the rest: $d^{\star}\left(\omega_{\beta_{j}}\right)=\omega_{\alpha_{i}}$ if $j=i 2^{n-m}$, in the opposite case $d^{\star}\left(\omega_{\beta_{j}}\right)=0$.

At any given scale we have an effective theory and we can calculate the expected values of every observable at this scale. For an observable $\hat{O}$ we consider its expectation value on normalized states $\kappa_{C_{n}}^{2}\left(\psi, o_{n} \psi\right)_{C_{n}}=o_{n}(\psi), o_{n}: \mathcal{H}_{n} \rightarrow \mathbb{R}$. The normalization factors in the inner product $\kappa_{C_{n}}^{2} \in \mathbb{R}^{+}$have to be adjusted in such a way that, at least in the continuum limit, the observables of different scales are pasted correctly by the decimation maps. ${ }^{2}$

\footnotetext{
${ }^{1}$ It is called coarse graining because these mappings induce a 'coarse graining' of the measure and the observables.

${ }^{2}$ In standard introductions the continuum limit in the renormalization group framework includes a wave function renormalization; here we choose the equivalent action of renormalizing the inner product instead. We choose to absorb the normalization factors in the inner product to find a non trivial action of the completely renormalized observable $\hat{O}$ in $\mathcal{H}_{\text {poly }, x}$.
} 
At a given scale $C_{m}$ we can "include the effects of more microscopic degrees of freedom" by using our decimation map. When $C_{m} \leq C_{n}$ we define

$$
o_{m(n)}:=d_{m, n}^{\star} O_{n} .
$$

That is, $o_{m(n)}\left(e_{\alpha_{i}}\right):=o_{n}\left(d_{m, n} e_{\alpha_{i}}\right)$. If these microscopically corrected observables converge, their limit will be called a completely renormalized observable at the given scale $o_{m}^{\text {ren }}: \mathcal{H}_{m} \rightarrow$ $\mathbb{R}$,

$$
o_{m}^{\text {ren }}:=\lim _{C_{n} \rightarrow \mathbb{R}} o_{m}(n) .
$$

By construction, when the completely renormalized observables exist, they are compatible with each other in the sense that $d_{m, n}^{\star} o_{n}^{\text {ren }}=o_{m}^{\text {ren }}$. A collection of compatible observables defines in itself a continuum limit observable.

A natural choice of normalization factors that leads to convergence in (2) for the case of the simple harmonic oscillator (SHO) [6] is $\kappa_{C_{n}}^{2}=2^{n}$ which means that the renormalized inner product in $\mathcal{H}_{n}^{\star}$ is $\left(\omega_{\alpha_{i}}, \omega_{\alpha_{j}}\right)_{C_{n}}^{\text {ren }}=\frac{1}{2^{n}} \delta_{i j}{ }^{3}$. The Hilbert space of covectors together with such inner product will be called $\mathcal{H}_{n}^{\star r e n}$. A sequence of covectors $\left\{\Psi_{C_{n}}^{\text {ren }} \in \mathcal{H}_{n}^{\star \text { ren }}\right\}$ is called compatible if $d^{\star} \Psi_{C_{n}}^{\text {ren }}=\Psi_{C_{m}}^{\text {ren }}$ for each pair with $m \leq n$. These compatible sequences form the Hilbert space $\overleftarrow{\mathcal{H}}_{\mathbb{R}}^{\star r e n}$ and the inner product in this space is $\left(\left\{\Psi_{C_{n}}\right\},\left\{\Phi_{C_{n}}\right\}\right)_{\mathbb{R}}^{\text {ren }}:=$ $\lim _{C_{n} \rightarrow \mathbb{R}}\left(\Psi_{C_{n}}, \Phi_{C_{n}}\right)_{C_{n}}^{\text {ren }}$. Observables in this continuum limit $o_{\mathbb{R}}^{\text {ren }}: \overleftarrow{\mathcal{H}}_{\mathbb{R}}^{\star \text { ren }} \rightarrow \mathbb{R}$ are defined by

$$
o_{\mathbb{R}}^{\mathrm{ren}}\left(\left\{\Psi_{C_{n}}\right\}\right):=\lim _{C_{n} \rightarrow \mathbb{R}} o_{n}\left(\left(\Psi_{C_{n}}, \cdot\right)_{C_{n}}^{\mathrm{ren}}\right) .
$$

The continuum limit of the effective theories exists if the above limit exists for enough observables of physical interest.

A physical Hilbert space can be defined when one considers the degeneracy of the inner product and the observables that we just defined. In the case of SHO it is shown that the corresponding physical Hilbert space is unitarily isomorphic to $L^{2}(\mathbb{R}, \mathrm{d} x)$, the usual Hilbert space of the Schrödinger theory [6] .

\section{LOOP QUANTUM COSMOLOGY}

In this section we shall follow [7] closely in order to define the model of interest. The gravitational phase space variables in the homogeneous, isotropic, spatially flat sector of general relativity can be expressed as,

$$
A_{a}^{i}=c V_{0}^{-(1 / 3) \mathrm{o}} \omega_{a}^{i} \quad \text { and } \quad E_{i}^{a}=p V_{0}^{-(2 / 3)}{\sqrt{q_{0}}}^{\circ} e_{i}^{a}
$$

where $V_{0}$ is the volume as given by the fiducial metric ${ }^{\circ} q_{a b}$ of an auxiliary cell that is used to make the integrals in the variational principle (and canonical formulation) of homogeneous

3 In the case of LQC the symmetric gravity plus matter constrained system is parametrized as an unconstrained system with one degree of freedom evolving in the relative clock of the matter. That parametrized system is then represented in a Hilbert space at "scale" $n$ which is similar to the $\mathcal{H}_{n}$ defined above, but whose inner product is not $\delta_{i j}$. A renormalized inner product for the dual Hilbert space would have to be defined following our procedure. 
models finite and ${ }^{\circ} \omega_{a}^{i}$ is the dual basis to a fiducial triad ${ }^{\circ} e_{i}^{a}$, compatible with ${ }^{\circ} q_{a b}$. In terms of geometrodynamical variables $|p|$ is proportional to $a^{2}$, where $a$ is the scale factor of the FRW metric, and $c$ is proportional to $\dot{a}$ (which determines the extrinsic curvature).

The fundamental Poisson bracket for the gravity variables is given by, $\{c, p\}=\frac{8 \pi G \gamma}{3}$, where $\gamma$ is the Barbero-Immirzi (BI) parameter that parametrizes an ambiguity in the loop quantization.

Defining the new coordinates,

$$
\mathrm{b}:=\frac{c}{|p|^{1 / 2}} \quad \text { and } \quad V:=p^{3 / 2}
$$

$V$ is the physical volume of the auxiliary cell. The new coordinate $\mathrm{b}$ is then equal to $\mathrm{b}=\frac{c}{a}$. On the constraint surface, that is, on classical solution to the equations of motion it

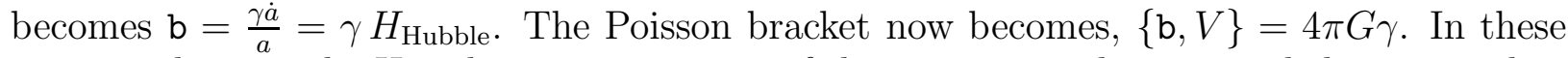
new coordinates, the Hamiltonian constraint of the gravitational part coupled to a massless scalar field $\phi$ is:

$$
\mathcal{C}:=-\frac{6}{\gamma^{2}} V \mathrm{~b}^{2}+8 \pi G \frac{p_{\phi}^{2}}{V}=0
$$

with $p_{\phi}$ the momentum conjugate to $\phi$. One has to define a corresponding operator $\hat{\mathcal{C}}$. But on the polymeric Hilbert space $\hat{b}$ is not well defined. In order to 'regulate it', the natural basis to consider is: $\left|\nu_{n}\right\rangle=|\lambda n\rangle$, with $\hat{V}\left|\nu_{n}\right\rangle=2 \pi \ell_{\mathrm{p}}^{2} \hat{\nu}\left|\nu_{n}\right\rangle=2 \pi \ell_{\mathrm{p}}^{2} \nu_{n}\left|\nu_{n}\right\rangle$ That is, the lattice has uniform spacing in the coordinate $\nu$ with spacing given by $\lambda$, the parameter that dictates the scale as understood in the previous section. Note that it is an eigenbasis of the volume operator. A wave function $\tilde{\Psi}(\nu)$ has only support on the discrete set $\nu_{n}=\lambda n$. Intuitively, in the regularization one is going to replace $b$ for: $\hat{b} \longrightarrow \frac{\widehat{\sin (\lambda \mathrm{b})}}{\lambda}$ (see [7] for the precise construction). The constraint is

$$
\partial_{\phi}^{2} \tilde{\Psi}(\nu, \phi)=3 \pi G|\nu| \frac{\sin \lambda \beta}{\lambda}|\nu| \frac{\sin \lambda \beta}{\lambda} \tilde{\Psi}(\nu, \phi)
$$

which takes the form [7]:

$$
\partial_{\phi}^{2} \tilde{\Psi}(\nu, \phi)=\frac{3 \pi G}{4 \lambda^{2}} \nu[(\nu+2 \lambda) \tilde{\Psi}(\nu+4 \lambda)-4 \nu \tilde{\Psi}(\nu)+(\nu-2 \lambda) \tilde{\Psi}(\nu-4 \lambda)]
$$

With the physical inner product given by

$$
(\tilde{\Psi}, \tilde{\Phi})_{\text {phy }}=\sum_{n \in \mathbb{Z}-0} \frac{1}{|4 n \lambda|} \tilde{\Psi}\left(\nu_{n}\right) \tilde{\Phi}\left(\nu_{n}\right) .
$$

The physical inner product (and Hilbert space) in this section refers to those objects that are needed in order to make physical predictions from the solutions of the quantum constraints for constrained systems (as is the case here). This has to be contrasted with the physical Hilbert space defined as an appropriate limit in [6].

Let us now take functions of $\Psi(\mathrm{b}, \phi)$. In this case the quantum constraint of sLQC (after a rescaling $\chi=\Psi / \nu)$, is given by,

$$
\frac{\partial^{2}}{\partial \phi^{2}} \cdot \chi(\mathrm{b}, \phi)=12 \pi G\left(\frac{\sin (\lambda \mathrm{b})}{\lambda} \frac{\partial}{\partial \mathrm{b}}\right)^{2} \cdot \chi(\mathrm{b}, \phi)
$$


with $\mathrm{b} \in(0, \pi / \lambda)$ and $\chi(\mathrm{b}, \phi)=-\chi(\pi / 2 \lambda-\mathrm{b}, \phi)$. Let us note that since the variable $\nu$ is discrete, its canonically conjugate variable $\mathrm{b}$ is compactified [8]. If we define a new $x$ coordinate as: $x=\frac{1}{\sqrt{12 \pi G}} \ln \left(\tan \left(\frac{\lambda \mathrm{b}}{2}\right)\right)$, then the basic constraint equation (9) translates to $\partial_{\phi}^{2} \chi(x, \phi)=\partial_{x}^{2} \chi(x, \phi)$. A general solution $\chi(x, \phi)$ to the previous equation can be decomposed in to left moving and right moving components: $\chi=F(\phi+x)-F(\phi-x)$ that furthermore, satisfy the required symmetry.

In SLQC the expectation value for $\hat{V}$, is given by:

$$
\left\langle\left.\hat{V}\right|_{\phi}\right\rangle=V_{+} e^{-\sqrt{12 \pi G} \phi}+V_{-} e^{\sqrt{12 \pi G} \phi}
$$

There is a minimum for the expectation value at the bounce time $\phi_{b}^{V}$ given by,

$$
\phi_{b}^{V}:=\frac{1}{2 \sqrt{12 \pi G}} \ln \left(\frac{V_{+}}{V_{-}}\right)
$$

On the other hand, the WDW equation in the $\mathrm{b}$ representation reads,

$$
\partial_{\phi}^{2} \cdot \chi(\mathrm{b}, \phi)=12 \pi G\left[\mathrm{~b} \partial_{\mathrm{b}}\right]^{2} \cdot \chi(\mathrm{b}, \phi)
$$

Introduce $y:=\frac{1}{\sqrt{12 \pi G}} \ln \left(\mathrm{b} / 2 \mathrm{~b}_{0}\right)$, from which the equation becomes,

$$
\partial_{\phi}^{2} \chi(y, \phi)=\partial_{y}^{2} \chi(y, \phi)
$$

The same equation as in LQC! What is the main difference then? Of course, the representation of the Dirac observables $\hat{V}_{\phi}$ :

$$
\text { WDW }: \quad \hat{\nu}:=\exp (\sqrt{12 \pi G} y) \partial_{y} \quad ; \quad \text { LQC }: \quad \hat{\nu}:=\cosh (\sqrt{12 \pi G} x) \partial_{x}
$$

Therefore, in the WDW theory

$$
\langle\hat{V}\rangle=\underline{V}_{0} e^{ \pm(\sqrt{12 \pi G} \phi)}
$$

with the sign depending on the choice of branch. For a given $\lambda$ we can have a WDW description in terms of the Klein Gordon equation where the choice of the parameter $b_{0}$ is rather convenient: set $\mathrm{b}_{0}=2 / \lambda$. In this case, if we fix a state $F\left(x_{+}\right)$for $\phi_{0}=0$ given, that corresponds to an initial state with support on small values of $x \ll-1$, we can expect it to have a small density (by fixing $p_{\phi}$ and having large volume). For that class of states, we shall compare the WDW and sLQC. The Hilbert space is the same, so the same wave function (solution to $\mathrm{KG}$ ) is a physical state on both theories, and the expectation value of the volume can be arbitrary close for both theories. The difference is given by [7],

$$
\left\langle\left.\hat{V}\right|_{\phi}\right\rangle_{\mathrm{wdw}}-\left\langle\left.\hat{V}\right|_{\phi}\right\rangle_{\lambda}=V_{-} e^{-\sqrt{12 \pi G} \phi}
$$

with $V_{-} \ll 1$ the initial difference, that goes to zero as $\lambda \rightarrow 0$. Note that this prescription is such that $\left[\left\langle\left.\hat{V}\right|_{\phi}\right\rangle_{\mathrm{wdw}}-\left\langle\left.\hat{V}\right|_{\phi}\right\rangle_{\lambda}\right] \rightarrow 0$ when $\phi \rightarrow \infty$, but that the difference grows unboundedly for the other sigh of $\phi$ (in the direction of the big bang).

This assignment that relates the 'discrete theory at scale $\lambda$ ' with WDW, the putative continuum theory, can also be used for defining a 'coarse graining map' between scales $\lambda$ and $\lambda^{\prime}=\alpha \lambda$ [7]: Given a state $\chi^{\lambda}(x, \phi)$ define the new state $\chi^{\lambda^{\prime}}(x, \phi)=\chi^{\lambda}(x+\mu, \phi)$ with 
$\mu=-\frac{1}{\sqrt{12 \pi G}} \ln \alpha$. This assignment ensures that the difference in volume at that time is very small: $\Delta(\langle\hat{V}\rangle)=(1-\alpha) V_{-} \ll 1$, since $V_{-} \ll 1$ and $\alpha<1$. Again, under time evolution, the difference will grow unboundedly as $\phi \rightarrow-\infty$ :

$$
\left.\Delta(\langle\hat{V}\rangle)\right|_{\phi}=(1-\alpha) V_{-} e^{-\sqrt{12 \pi G} \phi}
$$

Thus, with this prescription the time evolution is such that the physical observables become closer in the macroscopic domain (where the universe is large), and only depart when approaching the Planck domain to grow unboundedly on the pre big bang era [7]. It was also shown that there is no 'coarse graining map' for which the observable $\hat{p}_{\phi}$ (and any power of it) and the volume $\left.\hat{V}\right|_{\phi}$ can preserve their expectation values throughout the 'renormalization flow', for all times, preserving desired features in the large volume, macroscopic regime [7]. From this perspective one is lead to conclude that, as the scale $\lambda$ is refined, there is no limiting theory. Therefore, it is concluded that there is no continuum limit and thus sLQC is, in a precise sense, fundamentally discrete [7].

What we still need to understand is how this 'coarse graining map' [7] can be translated to the representation $\Psi(\nu)$ where the notion of scales and 'lattice refinement' is natural. As a second step, one needs to study the properties of the limiting Hilbert spaces and the action of the Hamiltonian of the theory that generates time evolution in $\phi$. We shall leave these matters for further investigation.

\section{DISCUSSION}

Ashtekar, Corichi and Singh introduced a solvable model [7] in order to study several aspects of loop quantum cosmology including its relation to the more traditional Wheeler DeWitt theory, a putative candidate for a continuum limit of LQC. Given that the model is completely solvable, it is a rich arena to study many aspects of loop quantum cosmology, polymer quantum mechanics and in general to study the physics of models based on nonregular representations of the Heisenberg-Weyl algebra. Their study of the continuum limit of the sLQC model, understood as approaching the limit in which the discrete nature of the quantum geometry is expected to go to the continuum, involved considering the behavior of the volume observable and its evolution (according to the internal clock defined by the scalar matter, and fixing the other observable of the theory). In the model there is not one single evolution operator, but one for each "auxiliary scale". The results of [7] show that, as the "auxiliary scale" is refined, the evolution of the volume observable does not converge to a hypothetical continuum theory (and therefore neither to the Wheeler DeWitt theory).

The work in [7] teaches us some aspects of the dynamics of the solvable loop quantum cosmology model, and about a specific 'coarse graining map' that is naturally suited to the formulation of the model as a solvable system. However, a detailed study of the model according to the framework of effective theories, coarse graining and continuum limit for polymer quantum mechanics as understood in [6], remains to be undertaken. In particular, the physical Hilbert space of the continuum as defined in [6] has not been explicitly constructed and the behavior of the observables and the dynamics thereon has yet to be studied. Work along this line is in progress.

A second and important question pertains to the conclusions one might draw from either result coming out of such investigations. In particular there might be very different expectations depending on some personal bias: For instance, some people may not find the 
notion of "scale" as natural in this case as it was in the case of polymer quantum mechanics. Therefore there may be several conclusions one may reach from the non existence of the limit studied in [7]. Here we show two lines of thinking: A) The system is fundamentally discrete and there is a "scale" $\lambda$ such that the dynamics encoded in the constraint of sLQC at that value of $\lambda$ is the fundamental dynamics. The constraints which use other values of the parameter are just incorrect; they are not effective theories that describe the system approximately. B) A "removal of the cut-off" by the limit studied in [7] is not the correct way to proceed. Instead, one may try to follow the lines of [6] in which at each scale one has an effective theory and at each such scale one can import corrections from smaller scale by a coarse graining procedure. The resulting mathematical structure of the limits taken in such an approach is different from those of [7] and needs to be studied.

If the continuum limit suggested by [6] does not exist, the coarse grained effective dynamics does not become better approximations to "the true system" as the scale is refined and thus either the effective dynamics at a given scale or the coarse graining procedure are wrong. In this case our procedure of non-perturbative renormalization would fail to define a theory of sLQC in the continuum. To understand which of these scenarios is realized is a pressing matter.

\section{Acknowledgments}

We thank A. Ashtekar and P. Singh for discussions. This work was partially supported by CONACyT U47857-F and NSF PHY04-56913 grants and by the Eberly Research Funds of Penn State.

[1] Ashtekar A, Lewandowski J 2004 Background independent quantum gravity: A status report Class. Quant. Grav. 21, R53 (2004); Rovelli C Quantum Gravity, (Cambridge, 2004); Thiemann T, Modern Canonical Quantum General Relativity, (Cambridge, 2007).

[2] Ashtekar A, Fairhurst S and Willis J L 2003 Quantum gravity, shadow states, and quantum mechanics Class. Quant. Grav. 201031 arXiv:gr-qc/0207106.

[3] Fleischhack C 2004 Representation of the Weyl algebra in quantum geometry arXiv:math-ph/0407006]; Lewandowski J, Okolow A, Sahlmann H and Thiemann T 2006 Uniqueness of diffeomorphism invariant states on holonomy-flux algebras Comm. Math. Phys. 267703 [arXiv:gr-qc/0504147v2]

[4] Ashtekar A 2007 An Introduction to Loop Quantum Gravity Through Cosmology Nuovo Cimento 112B, 1; Bojowald M 2005 Loop quantum cosmology Living Rev. Rel. 8, 11. arXiv:gr-qc/0601085;

[5] Manrique E, Oeckl R, Weber A and Zapata J A 2006 Loop quantization as a continuum limit Class. Quant. Grav. 233393 arXiv:hep-th/0511222;

[6] Corichi A, Vukasinac T and Zapata J A 2007 Hamiltonian and Physical Hilbert space in polymer quantum mechanics Class. Quant. Grav. 241 arXiv:gr-qc/0610072

[7] Ashtekar A, Corichi A and Singh P 2007 On the robustness of key features of loop quantum cosmology, arXiv:0710.3565 [gr-qc]].

[8] Corichi A, Vukasinac T and Zapata J A 2007 Polymer quantum mechanics and its continuum limit Phys. Rev. D 760440163 arXiv:0704.0007 